\title{
Successful Treatment of Peritoneal Dialysis-related Peritonitis due to Mycobacterium iranicum
}

\author{
Koji Inagaki ${ }^{1}$, Makoto Mizutani ${ }^{1}$, Yasuko Nagahara ${ }^{1}$, Marina Asano ${ }^{1}$, Daijiro Masamoto ${ }^{1}$, \\ Osamu Sawada ${ }^{2}$, Akio Aono ${ }^{3}$, Kinuyo Chikamatsu ${ }^{3}$ and Satoshi Mitarai ${ }^{3}$
}

\begin{abstract}
A 68-year-old man on peritoneal dialysis (PD) was hospitalized with the clinical picture of peritonitis. The patient was diagnosed with peritonitis caused by nontuberculous mycobacteria (NTM) according to positive Ziehl-Neelsen staining and negative Mycobacterium tuberculosis polymerase chain reaction results. Oral levofloxacin and clarithromycin, and later intraperitoneal imipenem were started. According to the anti-NTM susceptibility test results, oral minocycline was administered. The patient was treated for 6 months. He recovered without PD catheter removal; thus, PD was successfully continued. A genetic analysis identified the isolate as Mycobacterium iranicum. This is the first report of PD-related peritonitis caused by M. iranicum.
\end{abstract}

Key words: Mycobacterium iranicum, peritoneal dialysis, peritonitis

(Intern Med 55: 1929-1931, 2016)

(DOI: 10.2169/internalmedicine.55.5219)

\section{Introduction}

Peritoneal dialysis (PD)-related peritonitis is a major complication of PD therapy, and it is one of the most common reasons for PD withdrawal (1). When PD-associated peritonitis is culture negative, persistent or recurrent, or unresponsive to standard empirical antibiotic treatment, nontuberculous mycobacteria (NTM) should be suspected. We herein report a case of PD peritonitis caused by Mycobacterium iranicum, a rapidly growing mycobacterium that has not previously been linked with peritonitis. We successfully treated $M$. iranicum peritonitis without removing the PD catheter and discontinuing PD.

\section{Case Report}

A 68-year-old man with diabetic nephropathy had undergone PD for 7 months. The PD bag exchange protocol was $1 \mathrm{~L}$ exchanges of a $1.5 \%$ glucose-based solution (Dianeal-N PD-2 peritoneal dialysis solution with $1.5 \%$ dextrose; Baxter) four times daily. To change the PD fluid bags, the patient used a sterile connecting ultraviolet flash device. He was not treated with any immunosuppressive therapies, and a serological test for human immunodeficiency virus (HIV) was negative. He had no history of peritonitis and had not taken any antibiotics for 6 months. Interestingly, he frequently cultivated flowers.

Upon visiting our hospital, the patient presented with cloudy peritoneal fluid. Aside from this, he did not have any symptoms such as abdominal pain or a fever. His vital signs were as follows: blood pressure, 138/81 $\mathrm{mmHg}$; pulse, 89 / $\mathrm{min}$; and temperature, $36.6^{\circ} \mathrm{C}$. On physical examination, no abdominal tenderness was found, and the peritoneal catheter exit site was clear. The blood laboratory data were as follows: total WBC count, 8,100 cells $/ \mathrm{mm}^{3}$ with $69.1 \%$ neutrophils, $17.6 \%$ lymphocytes, $9.0 \%$ monocytes, $3.7 \%$ eosinophils, and $0.6 \%$ basophils; blood urea, $41.1 \mathrm{mg} / \mathrm{dL}$; plasma creatinine, $6.99 \mathrm{mg} / \mathrm{dL}$; C-reactive protein, $1.9 \mathrm{mg} / \mathrm{dL}$; and HbA1c, 5.4\%. The WBC count of the peritoneal fluid was 1,200 cells $/ \mathrm{mm}^{3}$ with $55 \%$ neutrophils and $45 \%$ lymphocytes.

On first presentation, he was hospitalized. An intraperitoneal infusion of cefazolin and ceftazidime was administered. On day 2, the dialysate that was obtained at admission showed a positive Ziehl-Neelsen stain and negative Мyco-

${ }^{1}$ Department of Nephrology, Handa City Hospital, Japan, ${ }^{2}$ Department of Clinical Laboratory, Handa City Hospital, Japan and ${ }^{3}$ Department of Mycobacterium Reference and Research, Research Institute of Tuberculosis, Japan Anti-tuberculosis Association, Japan

Received for publication February 24, 2015; Accepted for publication October 12, 2015

Correspondence to Dr. Koji Inagaki, inakou17@yahoo.co.jp 
Table. Results of Anti-rapidly Growing Nontuberculous Mycobacteria Susceptibility Testing of Mycobacterium iranicum Isolated from the Peritoneal Fluid. This was Enforced in Compliance with the CLSI-M24-A2 Guidelines.

\begin{tabular}{ccc}
\hline Antimicrobial agent & MICs $(\mu \mathrm{g} / \mathrm{mL})$ & Susceptible $(\mu \mathrm{g} / \mathrm{mL})$ \\
\hline Amikacin & 1 & $<$ or $=16$ \\
Clarithromycin & 0.5 & $<$ or $=2.0$ \\
Ciprofloxacin & 0.06 & $<$ or $=1.0$ \\
Imipenem & 0.25 & $<$ or $=4.0$ \\
Minocycline & 0.25 & $<$ or $=1.0$ \\
\hline
\end{tabular}

CLSI: Clinical Laboratory and Standards Institute

MICs: minimal inhibitory concentrations

bacterium tuberculosis polymerase chain reaction (PCR) results. The patient was diagnosed with PD-related peritonitis caused by NTM. Oral levofloxacin (500 mg every second day) and clarithromycin (400 mg daily) were administered. On day 5, the WBC count of the peritoneal fluid increased to 13,200 cells $/ \mathrm{mm}^{3}$; thus, the previous intraperitoneal antibiotics were discontinued. Subsequently, intraperitoneal imipenem (1,000 mg daily) was administered. On day 12, the peritoneal fluid became clear, and the WBC count of the peritoneal fluid decreased to 900 cells $/ \mathrm{mm}^{3}$. Drug minimal inhibitory concentrations (MICs) were determined using the broth microdilution method per the Clinical Laboratory and Standards Institute (CLSI) guidelines (2). The NTM was susceptible to amikacin, ciprofloxacin, clarithromycin, imipenem, and minocycline (Table). According to the susceptibility test results, intraperitoneal imipenem was discontinued, and oral minocycline (100 mg daily) was introduced on day 51. The WBC count of the peritoneal fluid decreased to $<100$ cells $/ \mathrm{mm}^{3}$, and the patient was discharged on day 55. After discharge, all three oral antibiotics were continued for 4 months. At the time of writing, 3 months after the cessation of therapy, the patient has continued PD without relapsing peritonitis.

Although, the dialysate showed a positive acid-fast culture on day 5, we were not able to identify the type of NTM. Further identification of the isolate was performed at the Research Institute of Tuberculosis, Japan Anti-tuberculosis Association on day 25. 16S rRNA and hsp65 sequencing were performed, and the results showed a $100 \%$ and $99.2 \%$ sequence identity with $M$. iranicum, respectively, on day 38 .

\section{Discussion}

PD-related peritonitis caused by NTM is rare, with an incidence of approximately 1/1,000 PD patients per-year (3). NTM is classified as either rapid or slow growing according to its capacity to form colonies before or after 7 days of culture, respectively. Most cases of NTM peritonitis have been linked to rapidly growing NTM, such as $M$. fortuitum, M. chelonae, and M. abscessus (4).

M. iranicum was described in 2013 on the basis of eight clinical strains isolated in six countries (Iran, Italy, Greece, the Netherlands, Sweden, and the United States). It is a rapidly growing scotochromogenic species with a yelloworange pigment. Based on a few housekeeping genes, a phylogenetic analysis showed that $M$. iranicum is closely related to another rapid grower, M. gilvum (5). Ten cases of $M$. iranicum infection have been reported, including pneumonia, meningitis, and soft tissue infection (5-7). However, its implications with PD-related peritonitis have not yet been reported.

Because treatment delay is a significant factor for mortality in patients with mycobacterial peritonitis (8), an early diagnosis is important. However, the early diagnosis of mycobacterial peritonitis in PD patients is very difficult because the symptoms and signs are indistinguishable from other bacterial peritonitis (4). The International Society for Peritoneal Dialysis guidelines emphasize that, in culture-negative peritonitis cases where empiric antibacterial therapy has failed, physicians should actively evaluate the peritoneal fluid for uncommon pathogens such as mycobacteria, fungi, and Nocardia (9). The diagnosis of PD-related peritonitis in the present case was based on the examination of an acidfast smear and culture.

NTM is found in the soil and water. The patient had washed the peritoneal catheter exit site with tap water, and exchanged the dialysate without adequately washing his hands after he had cultivated flowers. We presumed the cause of PD-related peritonitis was his contact with contaminated soil or water. We instructed him to wear gloves during gardening and wash his hands after gardening; however, because the patient wished to continue washing the peritoneal catheter exit site with tap water, this was allowed. Therefore, monitoring for relapse of the peritonitis was required.

In terms of rapidly growing NTM infection by nonpulmonary disease, it has been recommended that at least two antibiotics to which the organism is sensitive be given for a prolonged period (10). However, the removal of the PD catheter remains controversial. According to the report by Song et al., 52 of 56 affected patients had their PD catheters removed because they were unable to recover from peritonitis, irrespective of empirical antibacterial or anti-NTM therapies (4). Jiang et al. reported that 11 of 12 patients recovered from PD-related peritonitis caused by NTM; however, only three of the patients who recovered did so without removing their PD catheters (3). There might be two reasons for the recovery in our patient without catheter removal. First, we diagnosed PD-related peritonitis caused by NTM within 2 days because we examined the dialysate acid-fast smear and culture at the patient's first presentation. Therefore, we were able to start the appropriate antibiotic treatment early. Second, M. iranicum is susceptible to many antibiotics.

In summary, this is the first report of PD-related peritonitis caused by $M$. iranicum. At first presentation, we considered a potential mycobacterial infection and ordered a dialysate acid-fast culture. Therefore, we diagnosed the NTM infection early, and successfully treated the infection without 
removing the $\mathrm{PD}$ catheter. The patient was allowed to continue PD.

The authors state that they have no Conflict of Interest (COI).

\section{References}

1. Davenport A. Peritonitis remains the major clinical complication of peritoneal dialysis: the London, UK, peritonitis audit 20022003. Perit Dial Int 29: 297-302, 2009.

2. Woods GL, Brown-Elliot BA, Conville PS, et al. Susceptibility testing of mycobacteria, nocardiae and other aerobic actinomycetes. In: Approved Standard, 2nd ed. M24-A2. Clinical and Laboratory Standards Institute, Wayne, PA, 2011: 1-61.

3. Jiang SH, Roberts DM, Clayton PA, Jardine M. Non-tuberculous mycobacterial PD peritonitis in Australia. Int Urol Nephrol 45: 1423-1428, 2013.

4. Song Y, Wu J, Yan H, Chen J. Peritoneal dialysis-associated nontuberculous mycobacterium peritonitis: a systematic review of reported cases. Nephrol Dial Transplant 27: 1639-1644, 2012.
5. Shojaei H, Daley C, Gitti Z, et al. Mycobacterium iranicum sp. nov., a rapidly growing scotochromogenic species isolated from clinical specimens on three different continents. Int J Syst Evol Microbiol 63: 1383-1389, 2013.

6. Hashemi-Shahraki A, Heidarieh P, Azarpira S, Shojaei H, Hashemzadeh M, Tortoli E. Mycobacterium iranicum infection in HIV-infected patient, Iran. Emerg Infect Dis 19: 1696-1697, 2013.

7. Balakrishnan N, Tortoli E, Engel SL, Breitschwerdt EB. Isolation of a novel strain of Mycobacterium iranicum from a woman in the United States. J Clin Microbiol 51: 705-707, 2013.

8. Talwani R, Horvath JA. Tuberculous peritonitis in patients undergoing continuous ambulatory peritoneal dialysis: case report and review. Clin Infect Dis 31: 70-75, 2000.

9. Li PK, Szeto CC, Piranio B, et al. Peritoneal dialysis-related infections recommendations: 2010 update. Perit Dial Int 30: 393423, 2010 .

10. Griffith DE, Aksamit T, Brown-Elliott BA, et al. An official ATS/ IDSA statement: diagnosis, treatment, and prevention of nontuberculous mycobacterial diseases. Am J Respir Crit Care Med 175: 367-416, 2007.

(C) 2016 The Japanese Society of Internal Medicine http://www.naika.or.jp/imonline/index.html 